\title{
Colored partitions and a generalization of the braid arrangement
}

\author{
VOLKMAR WELKER ${ }^{1}$ \\ Fachbereich 6, Mathematik \\ Universität GH-Essen \\ D-45117 Essen, Germany \\ welker@exp-math.uni-essen.de
}

Submitted: November 11, 1996; Accepted: November 22, 1996.

\begin{abstract}
We study the topology and combinatorics of an arrangement of hyperplanes in $\mathbf{C}^{n}$ that generalizes the classical braid arrangement. The arrangement plays in important role in the work of Schechtman \& Varchenko [12, Part II] on Lie algebra homology, where it appears in a generic fiber of a projection of the braid arrangement. The study of the intersection lattice of the arrangement leads to the definition of lattices of colored partitions. A detailed combinatorial analysis then provides algebro-geometric and topological properties of the complement of the arrangement. Using results on the character of $S_{n}$ on the cohomology of these arrangements we are able to deduce the rational cohomology of certain spaces of polynomials in the complement of the standard discriminant that have no root in the first $s$ integers.
\end{abstract}

\section{Introduction}

In this paper we study the arrangement $\mathcal{A}_{n}^{\text {col,s }}$ of all affine hyperplanes $H_{i j}: z_{i}=z_{j}$, $1 \leq i<j \leq n$, and $H_{i}^{r}: z_{i}=r, 1 \leq i \leq n$ and $1 \leq r \leq s$. This arrangement appears in the work of Schechtman \& Varchenko [12, Part II] as a generic fiber of projections of the braid space in the context of Lie-algebra homology. We investigate the combinatorics of the intersection lattice $L_{\mathcal{A}_{n}^{\text {col,s }}}$ of $\mathcal{A}_{n}^{\text {col,s }}$ (i.e., the set of all subspaces that are intersections of hyperplanes in the arrangement, ordered by reversed inclusion). This leads to the definition of "colored partitions." Via the analysis of the homology of the order complex of the intersection lattice and using a formula by Orlik \& Solomon 10

\footnotetext{
${ }^{1}$ Supported by the DFG through "Habiliationsstipendium" We 1479/3

Keywords: Partition, hyperplane arrangement, intersection lattice, configuration space

Mathematics Subject Classification. Primary 05C40, 52B30. Secondary 05C40, 05E25
} 
we are able to determine the cohomology of the complement $\mathbf{C}^{n} \backslash \bigcup_{H \in \mathcal{A}_{n}^{\text {col,s }}} H$. The symmetric group $S_{n}$ acts on $\mathbf{C}^{n}$ by permuting the coordinates and leaving $\mathcal{A}_{n}^{\text {col,s }}$ invariant. By a calculation of the character of $S_{n}$ on the homology of the order complex of $L_{\mathcal{A}_{n}^{\text {cols }}}$ and using a formula of Orlik \& Solomon [10] we are able to describe the character of $S_{n}$ on the cohomology of the complement $\mathbf{C}^{n} \backslash \bigcup_{H \in \mathcal{A}_{n}^{\text {col,s }}} H$. Passing to rational cohomology and computing the space of $S_{n}$-invariants on the cohomology allows then a description of the rational cohomology of the quotient $\left(\mathbf{C}^{n} \backslash \bigcup H\right) / S_{n}$. The latter $H \in \mathcal{A}_{n}^{\text {col,s }}$

can then be identified with the space of polynomials $f(X)=X^{n}+a_{n-1} X^{n-1}+\cdots+a_{0}$ in $\mathbf{C}^{n}$ such that $f(X)$ has no double root and no root of $f(X)$ lies in $[s]:=\{1, \ldots, s\}$. These spaces can be used to approximate the space of monic polynomials of degree $n$ that have no double and no integral root.

\section{Basic Definitions}

An arrangement $\mathcal{A}$ of hyperplanes in $\mathbf{C}^{n}$ is a finite set of affine hyperplanes in complex $n$-space. To each arrangement $\mathcal{A}$ corresponds an $n$-dimensional complex manifold $M_{\mathcal{A}}=\mathbf{C}^{n} \backslash \bigcup_{H \in \mathcal{A}} H$. The space $M_{\mathcal{A}}$ is called the complement of the arrangement $\mathcal{A}$. The combinatorial object associated to an arrangement $\mathcal{A}$ is the intersection (semi)lattice $L_{\mathcal{A}}$. It is the set of subspaces $V$ of $\mathbf{C}^{n}$ such that $\emptyset \neq V=\bigcap_{H \in \mathcal{B}} H$ for some subset $\mathcal{B} \subseteq \mathcal{A}$ ordered by reversed inclusion. Here we allow $\mathcal{B}=\emptyset$ and identify the intersection $\bigcap_{H \in \emptyset} H$ with the space $\mathbf{C}^{n}$. Note, that in general $L_{\mathcal{A}}$ is actually not a lattice but a meet-semilattice (i.e., infima exist but suprema in general not). The link between the combinatorics of $L_{\mathcal{A}}$ and the topology of $M_{\mathcal{A}}$ is provided by the order complex of lower intervals in $L_{\mathcal{A}}$. In general, for a partially ordered set $P$ with top element $\hat{1}$ and least element $\hat{0}$ we denote by $\Delta(P)$ the order complex of $P$. This is the simplicial complex whose simplices are the chains $x_{0}<\cdots<x_{l}$ in $P \backslash\{\hat{0}, \hat{1}\}$. For $x \leq y, x, y \in P$, we write $[x, y]$ to denote the interval $\{z \mid x \leq z \leq y\}$ in $P$. If a finite subgroup $G \leq G l_{n}(\mathbf{C})$ acts on $\mathbf{C}^{n}$ leaving $M_{\mathcal{A}}$ invariant then $G$ also acts on $L_{\mathcal{A}}$ as a group of lattice automorphisms. If $V \in L_{\mathcal{A}}$ then the stabilizer $\operatorname{Stab}_{G}(V)=\left\{g \in G \mid V^{g}=V\right\}$ of $V$ in $G$ acts on the lower interval $[\mathbf{C}, V]$ in $L_{\mathcal{A}}$ as a group of lattice automorphisms. These actions induce a representation of $G$ on the cohomology of $M_{\mathcal{A}}$ and a representation of $\operatorname{Stab}_{G}(V)$ on the homology of the order complex $\Delta([\mathbf{C}, V])$. The following result by Orlik \& Solomon 10 links these two representations. 
Proposition 2.1 [10] Let $G \leq G l_{n}(\mathbf{C})$ be a finite group and let $\mathcal{A}$ be an arrangement of affine hyperplanes in $\mathbf{C}^{n}$ such that $M_{\mathcal{A}}$ is invariant under $G$. Then,

$$
\widetilde{H}^{i}\left(M_{\mathcal{A}}\right) \cong \bigoplus_{V \in L_{\mathcal{A}} / G \backslash\{\mathbf{C}\}} \operatorname{ind}_{S t a b_{G}(V)}^{G} \widetilde{H}_{\operatorname{codim}(V)-i-2}(\Delta([\mathbf{C}, V])),
$$

where $L_{\mathcal{A}} / G$ is a set of representatives of $G$-orbits on the lattice $L_{\mathcal{A}}$.

A result by Ziegler \& Živaljević [21] is concerned with the union $U_{\mathcal{A}}=\bigcup_{H \in \mathcal{A}} H$ of an arrangement $\mathcal{A}$. The result by Ziegler \& Živaljević is actually far more general and is valid for general arrangements of linear subspaces. Here, we state an equivariant version of the result by Ziegler \& Živaljević that can be found in [19].

Proposition 2.2[21] Let $\mathcal{A}$ be an arrangement of affine hyperplanes in $\mathbf{C}^{n}$. Assume $G \leq G l_{n}(\mathbf{C})$ is a finite subgroup that leaves $\mathcal{A}$ invariant. Let $\hat{1}$ be an additional element that is larger than any element of $L_{\mathcal{A}}$. Then, $U_{\mathcal{A}}$ is $G$-homotopy equivalent to $\Delta\left(L_{\mathcal{A}} \cup\{\hat{1}\}\right)$.

Based on these results, we start the investigation of the special class of arrangements we want to consider in this paper. Before we proceed, we recall a general method to determine the homotopy type of the order complex of a poset $P$. The formula is due to Björner \& Walker [3] for $G=1$ and can be found in [18] in the general case.

Lemma 2.3 [3] (Homotopy Complementation Formula) Let L be a (finite) lattice with least element $\hat{0}$ and largest element $\hat{1}$. Assume $G$ is a finite group of automorphisms of L. Let $a \in L \backslash\{\hat{0}, \hat{1}\}$ be a G-invariant element. Denote by $\operatorname{Co}(a)=$ $\{x \in L \mid \inf (x, a)=\hat{0}, \sup (x, a)=\hat{1}\}$ the set of complements of a. Then $\Delta(L)$ is G-homotopy equivalent to the wedge

$$
\bigvee_{x \in C o(a)} \operatorname{susp}(\Delta([\hat{0}, x]) * \Delta([x, \hat{1}]))
$$

where $G$ permutes the spaces in the wedge according to the action of $G$ on $L$.

In the formulation of the lemma we denote by "inf' the infimum operation in $L$ and by "sup" the supremum operation in $L$. We write " $\bigvee$ " for the wedge of topological spaces. Recall, that the wedge $X \vee Y$ of two topological is the disjoint union of $X$ and $Y$ modulo the identification of one point $x \in X$ with one point $y \in Y$. Note, that without specifying the points the wedge is (modulo homotopy) well defined whenever all spaces are path-connected. It turns out, that this is the case in the formula given by Lemma 2.3, except for some discrete 2-point spaces, where the wedge point has to be chosen to be one of the points. By "susp" we denote the suspension operation and by "*" we denote the join operation. Note, that in contrast to the common usage, we define the join of a space $X$ with the empty set to be the space $X$ itself and not the empty set. For more detailed information and the definitions we refer the reader to Munkres' book [9]. 


\section{A Generalization of the Braid Arrangement}

The classical braid arrangement $\mathcal{A}_{n}$ in complex $n$-space is given by the "thick" diagonals $H_{i j}: z_{i}=z_{j}$ for $1 \leq i<j \leq n$. The braid arrangement, also known as the complexified Coxeter arrangement of type $A$, is a well studied object (see for example Fox \& Neuwirth [7], Arnol'd [1], Brieskorn [4] and Lehrer \& Solomon [8]). Its name is derived from the fact that by a result of Fox \& Neuwirth [7] the complement $M_{\mathcal{A}_{n}}$ is the classifying space of the pure braid group on $n$ strings. We enlarge the central (i.e., all hyperplanes pass through the origin) arrangement $\mathcal{A}_{n}$ by some affine subspaces. Let $\mathcal{A}_{n}^{\text {col,s }}$ be the arrangement of complex hyperplanes $H_{i j}: z_{i}=z_{j}, 1 \leq i<j \leq n$ and $H_{i}^{r}: z_{i}=r$ for $1 \leq i \leq n, 1 \leq r \leq s$. This arrangement occurs in the work of Schechtman \& Varchenko [12, Part II]. More generally, Schechtman \& Varchenko 12] consider the projection of the complement $M_{\mathcal{A}_{n+s}}$ of the braid arrangement $\mathcal{A}_{n+s}$ of hyperplanes $H_{i j}: z_{i}=z_{j}, 1 \leq i<j \leq n+s$ in complex $(n+s)$-space on the last $s$ coordinates. Let $p r_{n, s}$ be the projection of $(n+s)$-space on the last $s$ coordinates. The image of $p r_{n, s}$ is complex $s$-space. For a point $\left(t_{1}, \ldots, t_{s}\right)$ in $p r_{n, s}\left(M_{\mathcal{A}_{n+s}}\right)$ (i.e., it satisfies $t_{i} \neq t_{j}$ for $\left.1 \leq i<j \leq s\right)$ the fiber $p r_{n, s}^{-1}\left(t_{1}, \ldots, t_{s}\right)$ of $p r_{n, s}$ when restricted to $M_{\mathcal{A}_{n+s}}$ is homeomorphic to $M_{\mathcal{A}_{n}^{\text {col,s. }}}$.

Let us define some combinatorial objects that turn out to be important in the investigation of the arrangement $\mathcal{A}_{n}^{\text {col,s }}$. We describe a partition $\tau$ of the set $[n]$ by $B_{1}|\cdots| B_{f}$, where $B_{i} \subseteq[n], B_{i} \cap B_{j}=\emptyset, \bigcup_{i=1}^{f} B_{i}=[n]$. The sets $B_{i}$ are called the blocks of $\tau$. We denote by $\Pi_{n}$ the lattice of all partition of $[n]$ ordered by refinement (i.e., we say $B_{1}|\ldots| B_{f} \leq C_{1}|\ldots| C_{e}$ if $f \leq e$ and for each $1 \leq i \leq f$ there is a $1 \leq j \leq e$ such that $\left.B_{i} \subseteq C_{j}\right)$. Let $\Pi_{n}^{\text {col,s }}, s \geq 1$, be the set of all pairs $(\tau=$ $\left.B_{1}|\cdots| B_{t},\left(l_{1}, \ldots, l_{t}\right)\right)$ of partitions $\tau \in \Pi_{n}$ and sequences of numbers $l_{i} \in\{0, \ldots, s\}$ of length $t$, where $t$ is the number of blocks of $\tau$ and for each $j \in[s]$ - note that then $j \neq 0$ - there is at most one index $i$ for which $l_{i}=j$. We say $\left(\tau=B_{1}|\cdots| B_{t},\left(l_{1}, \ldots, l_{t}\right)\right)$ is smaller than $\left(\tau^{\prime}=B_{1}^{\prime}|\cdots| B_{t^{\prime}}^{\prime},\left(l_{1}^{\prime}, \ldots, l_{t^{\prime}}^{\prime}\right)\right)$ if and only if $\tau \leq \tau^{\prime}$ and if $B_{i} \subseteq B_{j}^{\prime}$ then $l_{i} \neq 0$ implies $l_{j}^{\prime}=l_{i}$. We call an element $\left(\tau=B_{1}|\cdots| B_{t},\left(l_{1}, \ldots, l_{t}\right)\right)$ of $\Pi_{n}^{\text {col,s }}$ a colored partition of $[n]$. We call the number $l_{i}$ the color of the $i$ th block of $\tau$. The number " 0 " in this context stands for "no color." If $s=1$ then $\Pi_{n}^{\text {col,s }}$ is actually a lattice with top element $(|1 \cdots n|,(1))$. In general, let $\left(\tau,\left(l_{1}, \ldots, l_{t}\right)\right)$ and $\left(\tau^{\prime},\left(l_{1}^{\prime}, \ldots, l_{t^{\prime}}^{\prime}\right)\right)$ be two colored partitions. Assume $\left(\gamma,\left(m_{1}, \ldots, m_{q}\right)\right)$ is an upper bound of $\left(\tau,\left(l_{1}, \ldots, l_{t}\right)\right)$ and $\left(\tau^{\prime},\left(l_{1}^{\prime}, \ldots, l_{t^{\prime}}^{\prime}\right)\right)$. Then if $B_{i}$ is a block of $\tau$ (resp., $\left.\tau^{\prime}\right)$ then for the block $C_{j}$ of $\gamma$ that contains $B_{i}$ we have $m_{j}=0$ implies $l_{i}=0$ (resp., $l_{i}^{\prime}=0$ ). Hence, we may assume that if $B_{i} \subseteq C_{j}$ then $l_{i}=m_{j}$ (resp., $l_{i}^{\prime}=m_{j}$ ). We set $\tau^{\prime \prime}=\tau \vee \tau^{\prime}$ and for a block $D_{j}$ of $\tau^{\prime \prime}$ we set $n_{j}=l_{i}$ for any block $B_{i}$ of $\tau$ contained in $D_{j}$. Then $\left(\tau^{\prime \prime},\left(n_{1}, \ldots, n_{q^{\prime}}\right)\right)$ is an upper bound of $\left(\tau,\left(l_{1}, \ldots, l_{t}\right)\right)$ and $\left(\tau^{\prime},\left(l_{1}^{\prime}, \ldots, l_{t^{\prime}}^{\prime}\right)\right)$ that is smaller than $\left(\gamma,\left(m_{1}, \ldots, m_{q}\right)\right)$. Thus $\left(\tau^{\prime \prime},\left(n_{1}, \ldots, n_{q^{\prime}}\right)\right)$ is the supremum of $\left(\tau,\left(l_{1}, \ldots, l_{t}\right)\right)$ and $\left(\tau^{\prime},\left(l_{1}^{\prime}, \ldots, l_{t^{\prime}}^{\prime}\right)\right)$. In particular, this implies that all lower intervals in $\Pi_{n}^{\text {col,s }}$ are lattices.

Proposition 3.1 Let $1 \leq s, n$. The intersection lattice $L_{\mathcal{A}_{n}^{\text {col,s }}}$ is isomorphic to the partially ordered set of colored partitions $\Pi_{n}^{\text {col,s }}$.

Proof. Let $\left(\tau=\left(B_{1}|\ldots| B_{t}\right),\left(l_{1}, \ldots, l_{t}\right)\right)$ be a colored partition in $\Pi_{n}^{\text {col,s }}$. Then we map $\left(\tau=\left(B_{1}|\ldots| B_{t}\right),\left(l_{1}, \ldots, l_{t}\right)\right)$ to the affine subspace $V_{\left(\tau,\left(l_{1}, \ldots, l_{t}\right)\right)}$ that is defined by 
$z_{i}=z_{j}$ if $i$ and $j$ lie in the same block of $\tau$ and $z_{i}=l_{j}$ for $i \in B_{j}$ in case $l_{j} \neq 0$. Obviously, this is an order preserving map to $L_{\mathcal{A}_{n}^{\text {col,s. }}}$. Conversely, we map each element $V \in L_{\mathcal{A}_{n}^{\text {col,s }}}$ to the colored partition $\left(\tau=B_{1}|\ldots| B_{t},\left(l_{1}, \ldots, l_{t}\right)\right)$ that is defined by : $i, j$ lie in the same block of $\tau$ if $z_{i}=z_{j}$ and $l_{j}=z_{i}$ if $i \in B_{j}$ and $z_{i} \in[s]$. Obviously, the two maps are inverse to each other. One checks, that they induce indeed a poset isomorphism $L_{\mathcal{A}_{n}^{\mathrm{col}, \mathrm{s}}} \cong \Pi_{n}^{\mathrm{col}, \mathrm{s}}$.

A geometric semilattice is (see for example Wachs \& Walker [17]) a meet-semilattice $L$ that is constructed from a geometric lattice $L^{\prime}$ by removing an upper interval $[x, \hat{1}]$ for an atom $x$ of $L^{\prime}$ (i.e., $L=L^{\prime} \backslash[x, \hat{1}]$ ). If $L$ is a geometric semilattice then for each $x \in L$ the number of elements in a maximal chain from the least element $\hat{0}$ to $x$ is independent of the choice of the maximal chain. We denote by $\operatorname{rank}(x)$ the rank of $x$ in $L$ (i.e., the number of elements in a maximal chain in [0, $x]$ minus 1 ). As an immediate consequence we obtain :

Corollary 3.2 The partially ordered set $\Pi_{n}^{\mathrm{col}, \mathrm{s}}$ is a geometric semilattice. In particular, if $\hat{1}$ is an additional element and $s>1$ then the order complex $\Delta\left(\Pi_{n}^{\mathrm{col}, \mathrm{s}} \cup\{\hat{1}\}\right)$ is homotopic to a wedge of spheres of dimension $n-1$. For $s=1$ the complex $\Delta\left(\Pi_{n}^{\mathrm{col}, \mathrm{s}} \cup\{\hat{1}\}\right)$ is contractible. More generally, for an element $x \in \Pi_{n}^{\mathrm{col}, \mathrm{s}}$ the order complex of interval $[\hat{0}, x]$ is homotopic to a wedge of spheres of dimension rank $(x)-2$.

Proof. It is well known that the intersection lattice of an affine hyperplane arrangement is a geometric semilattice (see for example [1]). The corresponding geometric lattice can be constructed by enlarging the arrangement by a hyperplane at infinity and then considering the intersection lattice of the enlarged arrangement. By a result of Wachs \& Walker 17 the order complex $\Delta(L \cup\{\hat{1}\})$ of a geometric semilattice $L$ enhanced by an additional top element is homotopic to a wedge of spheres of dimensions $\operatorname{rank}(L \cup\{\hat{1}\})-2$. Also, for $x \in L$ the order complex of the interval $[\hat{0}, x]$ is homotopic to a wedge of spheres of dimension $\operatorname{rank}(x)-2$. Then the result for $s>1$ and for intervals follows from Proposition 3.1. It remains to treat the case $s=1$. As mentioned before in this case the (semi)lattice $\Pi_{n}^{\text {col,s }}$ has a top element (see also Remark 3.3 and Proposition 4.2). Thus the order complex of $\Pi_{n}^{\text {col,s }} \cup\{\hat{1}\}$ is a cone and hence contractible.

In order to give the reader a feeling for the combinatorial structure of the lattice of colored partitions, we classify the cover relations in $\Pi_{n}^{\mathrm{col}, \mathrm{s}}$. Let

$$
\left(\tau=B_{1}|\cdots| B_{t},\left(l_{1}, \ldots, l_{t}\right)\right)<\left(\tau^{\prime}=B_{1}^{\prime}|\cdots| B_{t^{\prime}}^{\prime},\left(l_{1}^{\prime}, \ldots, l_{t^{\prime}}^{\prime}\right)\right)
$$

be a cover relation in $\Pi_{n}^{\text {col,s }}$. Then either :

(A) $\tau=\tau^{\prime}$, there is a unique index $i$ such that $l_{j}=l_{j}^{\prime}$ for $j \neq i$ and $l_{i}=0, l_{i}^{\prime} \neq 0$.

(B) $\tau<\tau^{\prime}$ in $\Pi_{n}$ is a cover relation and $\tau^{\prime}$ is constructed from $\tau$ by merging the blocks $B_{i}$ and $B_{h}$ into the block $B_{j}^{\prime}$ for which $l_{i}=l_{h}=l_{j}^{\prime}=0$.

(C) $\tau<\tau^{\prime}$ in $\Pi_{n}$ is a cover relation and $\tau^{\prime}$ is constructed from $\tau$ by merging the blocks $B_{i}$ and $B_{h}$ into the block $B_{j}^{\prime}$ for which $l_{i}=l_{j}^{\prime} \neq 0$ and $l_{h}=0$. 
THE ELECTRONiC JOURNAL OF COMBINATORICS 4 (1997), \#R4

The following Remark 3.3 was first stated implicitly by Edelman \& Reiner [5]. They made the observation on the realm of arrangements that are extensions of the braid arrangement by some set of hyperplanes defined by equations $z_{i}= \pm z_{j}$ and $z_{i}=0$. Of course, this includes the arrangement $\mathcal{A}_{n}^{\text {col, } 1}$. The case $s>1$ is not considered by Edelman \& Reiner, their motivation for studying the corresponding arrangements origins in the "freeness" condition (see the book by Orlik \& Terao [11]) and therefore there is no further overlap with the work presented here.

Remark 3.3 The lattice $\Pi_{n}^{\mathrm{col}, 1}$ is $S_{n}$-isomorphic to $\Pi_{n+1}$.

Proof. We map a colored partition of $[n]$ to the partition of $[n+1]$ that is defined by adjoining $n+1$ to the colored block, in case there is one; or adjoining the singleton $\mid n+$ 1| in case there is no colored block. It is easily seen that this defines an $S_{n}$-equivariant $\left(S_{n}\right.$ regarded as the subgroup of $S_{n+1}$ stabilizing $\left.n+1\right)$ lattice isomorphism.

\section{Combinatorics \& Homology of Lattices of Col- ored Partitions}

In this section we determine the $G$-homotopy type of the posets $\left[\hat{0},\left(\tau,\left(l_{1}, \ldots, l_{t}\right)\right)\right]$ where $G$ is the stabilizer of $\left(\tau,\left(l_{1}, \ldots, l_{t}\right)\right)$ in $S_{n}$. First, we consider the structure of intervals $\left[\hat{0},\left(\tau=B_{1}|\cdots| B_{t},\left(l_{1}, \ldots, l_{t}\right)\right)\right]$. After possibly renumbering the blocks we may assume that $l_{f}=\cdots=l_{t}=0$ and $l_{1}, \ldots, l_{f-1} \neq 0$.

Lemma 4.1 Let $G$ be the stabilizer of the colored partition $\left(\tau=B_{1}|\cdots| B_{t},\left(l_{1}, \ldots, l_{t}\right)\right)$ in $S_{n}$. Assume that $l_{f}=\cdots=l_{t}=0$ and $l_{1}, \ldots, l_{f-1} \neq 0$. The interval $[\hat{0},(\tau=$ $\left.\left.B_{1}|\cdots| B_{t},\left(l_{1}, \ldots, l_{t}\right)\right)\right]$ is $G$-isomorphic to

$$
\times_{i=1}^{f-1} \Pi_{\left|B_{i}\right|}^{\mathrm{col}, 1} \times \times_{i=f}^{t} \Pi_{\left|B_{i}\right|} \cong \times_{i=1}^{f-1} \Pi_{\left|B_{i}\right|+1} \times \times_{i=f}^{t} \Pi_{\left|B_{i}\right|} .
$$

Proof. The isomorphism to the poset on the left hand side is obvious, since all blocks can be split independently. The second isomorphism then follows from Remark 3.3.

By the previous lemma it suffices to consider the $S_{n}$-lattices $\Pi_{n}^{\text {col,1 }}$ in order to understand the $G$-homotopy type of lower intervals in $\Pi_{n}^{\text {col,s }}$.

Proposition 4.2 The $S_{n}$-homotopy type of $\Pi_{n}^{\text {col,1 }}$ is given by a wedge of $n$ ! spheres of dimension $(n-2)$. The $n$ ! spheres are permuted by $S_{n}$ according to its regular representation. In particular, $\widetilde{H}_{n-2}\left(\Pi_{n}^{\text {col, } 1}\right)$ is the regular $S_{n}$-module.

Proof. Let $(|1 \cdots n|,(0))$ be the maximal element in $\Pi_{n}^{\text {col, } 1}$ with no colored block If a colored partition $\left(\tau,\left(l_{1}, \ldots, l_{t}\right)\right)$ is a complement of $(|1 \cdots n|,(0))$ then at least one (and therefore exactly one) index $i$ must satisfy $l_{i}=1$. Moreover, if there is a non-trivial block in $\tau$ then $(\tau,(0, \ldots, 0))$ is a lower bound for $(|1 \cdots n|,(0))$ and $\left(\tau,\left(l_{1}, \ldots, l_{t}\right)\right)$. Thus any complement of $(|1 \cdots n|,(0))$ must be of the form 
$(|1| \cdots|n|,(0, \ldots, \underbrace{1}_{i}, \ldots 0))$ where the 1 is at the $i$ th position. Hence there are $n$ complements of $(|1 \cdots n|,(0))$ and they are permuted by $S_{n}$ according to the natural $S_{n}$-action and each complement is stabilized by one of the one-point stabilizers $S_{n-1}$ in $S_{n}$. Each complement is an atom in $\Pi_{n}^{\text {col,1 }}$ and the upper intervals $[(|1| \cdots|n|,(0, \ldots, \underbrace{1}_{i}, \ldots 0)), \hat{1}]\left(\hat{1}\right.$ being the largest element $(|1 \cdots n|,(1))$ of $\left.\Pi_{n}^{\mathrm{col}, 1}\right)$

are $S_{n-1}$-isomorphic to $\Pi_{n} \cong \Pi_{n-1}^{\text {col,1 }}$. By the $G$-equivariant Homotopy Complementation Formula 2.3 the result follows.

Let us denote by $\mathbf{r}_{n}$ the character of the regular $S_{n}$-representation, by $\mathbf{s g n}_{n}$ the character of the sign-representation of $S_{n}$ and by $\mathbf{1}_{n}$ the character of the trivial $S_{n^{-}}$ representation. By $\pi_{n}$ we denote the character of $S_{n}$ on the homology of the order complex of $\Pi_{n}$ in dimension $n-3$. It is a well studied character of dimension $(n-1)$ ! (see Stanley [13 for a detailed description).

Corollary 4.3 Let $G$ be the stabilizer of $\left(\tau=B_{1}|\cdots| B_{t},\left(l_{1}, \ldots, l_{t}\right)\right)$ in $S_{n}$. Assume that $l_{f}=\cdots=l_{t}=0$ and $l_{1}, \ldots, l_{f-1} \neq 0$. Let $B_{f}|\cdots| B_{t}$ be a partition of type $\left(1^{e_{1}}, \ldots, n^{e_{n}}\right)$. Then

$$
G \cong S_{\left|B_{1}\right|} \times \cdots \times S_{\left|B_{f-1}\right|} \times S_{e_{1}}\left[S_{1}\right] \times \cdots \times S_{e_{n}}\left[S_{n}\right]
$$

The $S_{n}$-character on

$$
\operatorname{ind}_{G}^{S_{n}} \widetilde{H}_{n-t+f-2}\left(\left[\hat{0},\left(\tau,\left(l_{1}, \ldots, l_{t}\right)\right)\right]\right)
$$

is given by

$$
\operatorname{ind}_{G}^{S_{n}} \mathbf{r}_{\left|B_{1}\right|} \cdots \mathbf{r}_{\left|B_{f-1}\right|} \cdot \operatorname{sgn}_{e_{1}}\left[\pi_{1}\right] \cdot \mathbf{1}_{e_{2}}\left[\pi_{2}\right] \cdots
$$

Proof. The assertion follows immediately from Proposition 4.2 and the 16 , Theorem 1.1].

We are grateful to Richard Stanley for pointing out that the characteristic polynomial (see [14]) of $\Pi_{n}^{\text {col,s }}$ can be easily computed using a result about characteristic polynomials of hyperplane arrangements (see Orlik \& Terao [11, Theorem 2.69]) or more generally subspace arrangements (Athanasiadis [2, Theorem 2.2]). The characteristic polynomial $\chi(P, t)$ of a poset $P$ with rank function rank and minimal element $\hat{0}$ is defined by

$$
\chi(P, t)=\sum_{x \in P} \mu(\hat{0}, x) t^{\operatorname{rank}(P)-\operatorname{rank}(x)} .
$$

Here, $\operatorname{rank}(P)$ is the maximal rank of one of the elements of $P$ and " $\mu$ " denotes the Möbius function of $P$ (see 14]).

Proposition 4.4 Let $\mathcal{A}$ be an affine hyperplane arrangement in $\mathbf{C}^{n}$ such that the subspaces in $\mathcal{A}$ can be defined by equations using only integer coefficients. Let $\mathbf{F}_{q}$ denote the field with $q$ elements, $q$ a prime. By our assumption we then can regard $\mathcal{A}$ as an arrangement in $\mathbf{F}_{q}^{n}$. Then for large enough $q$ we have

$$
\chi\left(L_{\mathcal{A}}, q\right)=\left\|\mathbf{F}_{q}^{n} \backslash\left(\bigcup_{H \in \mathcal{A}} H\right)\right\| .
$$


Corollary 4.5 The characteristic polynomial $\chi\left(\Pi_{n}^{\mathrm{col}, \mathrm{s}}, q\right)$ is given by

$$
(q-s) \cdots(q-s-n+1) \text {. }
$$

Proof. If $\left(x_{1}, \ldots, x_{n}\right)$ is a point in the complement $\mathbf{F}_{q}^{n} \backslash\left(\bigcup_{H \in \mathcal{A}} H\right)$ then if $q$ is large enough there are $(q-s-(i-1))$ choices for the $i$ th coordinate $x_{i}$. From this observation, the result follows from the preceding Proposition 4.4 and Proposition 3.1.

So far we have treated lower intervals in $\Pi_{n}^{\text {col,s }}$. Now we turn our interest to $\Pi_{n}^{\text {col,s }}$ itself. Let us denote by $\hat{1}$ an additional element that is larger than all elements of $\Pi_{n}^{\text {col,s }}$. Then by standard facts about the characteristic polynomial (see [14]) the preceding proposition immediately implies that $\mu\left(\Pi_{n}^{\mathrm{col}, \mathrm{s}} \cup\{\hat{1}\}\right)=\chi\left(\Pi_{n}^{\mathrm{col}, \mathrm{s}}, 1\right)=$ $(-1)^{n}(s-1) \cdots((s-1)+(n-1))$.

Proposition 4.6 The poset $\Pi_{n}^{\mathrm{col}, \mathrm{s}} \cup\{\hat{1}\}$ is homotopy equivalent to a wedge of

$$
(s-1) \cdots((s-1)+(n-1))
$$

spheres of dimension $n-1$. The $S_{n}$-homotopy type of $\Pi_{n}^{\text {col,s }} \cup\{\hat{1}\}$ is a wedge of $n$ ! copies of a wedge of $\frac{(n+s-2) !}{(s-2) ! n !}$ spheres of dimension $n-1$, that are permuted according to the regular $S_{n}$-representation. In particular, if $s=1$ then $\Pi_{n}^{\text {col,s }} \cup\{\hat{1}\}$ is contractible. The representation of $S_{n}$ on $\widetilde{H}_{n}\left(\Pi_{n}^{\mathrm{col}, \mathrm{s}}\right)$ is given by $\frac{(n+s-2) !}{(s-2) ! n !}$ copies of the regular representation of $S_{n}$.

Proof. We give the non-equivariant part of the assertion. The equivariant part of the assertion follows using Proposition 2.2 from Theorem 5.1 (ii). Note, that in the proof of Theorem 5.1 (ii) we use the non-equivariant part of this Proposition 4.6.

By results of Wachs \& Walker [17 the order complex of a geometric semilattice $L$ enlarged by an additional top element $\hat{1}$ is homotopic to a wedge of spheres of dimension $\operatorname{rank}(L \cup\{\hat{1}\})-2$. In particular, the homology of the order complex is free of rank equal to the number of spheres and concentrated in one dimension. Since the Möbius number of a poset equals by a result of P. Hall (see for example [14]) the alternating sum of ranks of homology groups of the order complex of $P$, the result follows from the previous observations about the Möbius number.

\section{Geometry and Topology of the Arrangement}

Using results on the combinatorics of $\mathcal{A}_{n}^{\text {col,s }}$ presented in the preceding section, we obtain: 
Theorem 5.1 Let $1 \leq s, n$.

(i) There is an isomorphism of $S_{n}$-modules

$$
\widetilde{H}^{i}\left(\mathbf{C}^{n} \backslash U_{\mathcal{A}_{n}^{\text {col,s }}}\right) \cong \bigoplus_{p \in \Pi_{n}^{\mathrm{col}, \mathrm{s}} / S_{n} \backslash\{\hat{0}\}} \operatorname{ind}_{\operatorname{Stab}_{S_{n}}(p)}^{S_{n}} \widetilde{H}_{\operatorname{codim}\left(V_{p}\right)-i-2}(\Delta(\hat{0}, p)),
$$

where $V_{p}$ is the subspace in $L_{\mathcal{A}_{n}^{\text {col,s }}}$ corresponding to $p \in \Pi_{n}^{\text {col,s. }}$. In particular, $\widetilde{H}^{*}\left(\mathbf{C}^{n} \backslash U_{\mathcal{A}_{n}^{\text {col,s }}}\right)$ is free.

(ii) If $s>1$ then $U_{\mathcal{A}_{n}^{\text {col,s }}}$ is $S_{n}$-homotopic to a wedge of $n$ ! copies of a wedge of $\frac{(n+s-2) !}{(s-2) ! n !}$ spheres of dimension $n-1$, where the $n$ ! spaces are permuted according to the regular representation of $S_{n}$. In particular, the space $U_{\mathcal{A}_{n}^{\text {col,s }}} / S_{n}$ is homotopic to a wedge of $\frac{(n+s-2) !}{(s-2) ! n !}$ spheres of dimension $n$. If $s=1$ then $U_{\mathcal{A}_{n}^{\text {col,s }}}$ is $S_{n}$-contractible.

Proof. Part (i) follows immediately from Proposition 3.1, Proposition 2.1.

The proof of part (ii) is more subtle. If $s=1$ then the arrangement $\mathcal{A}_{n}^{\text {col,s }}$ is equivalent to a central arrangement (consider the point $(1, \ldots, 1)$ as the origin). The map sending all points in $U_{\mathcal{A}_{n}^{\text {col,s }}}$ to the origin defines an $S_{n}$-deformation retraction. In particular, $U_{\mathcal{A}_{n}^{\text {cols }}}$ is $S_{n}$-contractible (it is a general well known fact that the union of a central arrangement is contractible). Now consider the case $s>1$. By Proposition 2.2 we have $U_{\mathcal{A}_{n}^{\text {col,s }}} \simeq_{S_{n}} \Delta\left(\Pi_{n}^{\text {col,s }} \cup\{\hat{1}\}\right)$. Thus it suffices to consider the $S_{n}$-homotopy type of $U_{\mathcal{A}_{n}^{\text {col,s }}}$. Let us regard $\mathbf{R}^{n}$ as the subspace of $\mathbf{C}^{n}$ defined by the equations $\operatorname{Im}\left(z_{i}\right)=0$, $1 \leq i \leq n$. Then $U_{\mathcal{A}_{n}^{\mathrm{col}, \mathrm{s}}}^{\mathbf{R}}:=U_{\mathcal{A}_{n}^{\mathrm{col}, \mathrm{s}}} \cap \mathbf{R}^{n}$ is an $S_{n}$-deformation retract of $U_{\mathcal{A}_{n}^{\mathrm{col}, \mathrm{s}} \text {. The }}$ homotopy is given by $K: U_{\mathcal{A}_{n}^{\text {col,s }}} \times[0,1] \rightarrow U_{\mathcal{A}_{n}^{\text {col,s }}}$ that sends $\left(\left(x_{1}+i y_{1}, \ldots, x_{n}+i y_{n}\right), t\right)$ to $\left(x_{1}+i t y_{1}, \ldots, x_{n}+i t y_{n}\right)$. Actually this is a well known general fact about arrangements and their complexifications (see [21]). Let $U_{\mathcal{A}_{n}}^{\mathbf{R}}$ be the "real part" $U_{\mathcal{A}_{n}} \cap \mathbf{R}^{n}$ of union of the braid arrangement $\mathcal{A}_{n}=\left\{H_{i j}: z_{i}=z_{j} \mid 1 \leq i<j \leq n\right\}$. Then $U_{\mathcal{A}_{n}}^{\mathbf{R}}$ is a $S_{n^{-}}$ invariant subspace of $U_{\mathcal{A}_{n}^{\text {col, s }}}^{\mathbf{R}}$. Moreover, $U_{\mathcal{A}_{n}}^{\mathbf{R}}$ is $S_{n}$-contractible. The map $L: U_{\mathcal{A}_{n}}^{\mathbf{R}} \times$ $[0,1] \rightarrow U_{\mathcal{A}_{n}}^{\mathbf{R}}$ defined by $L\left(\left(z_{1}, \ldots, z_{n}\right), t\right)=t \cdot\left(z_{1}, \ldots, z_{n}\right)$ defines a $S_{n}$-homotopy from $i d_{U_{\mathcal{A}_{n}}^{\mathbf{R}}}$ to the constant map from $U_{\mathcal{A}_{n}}^{\mathbf{R}}$ to the origin $\underline{\mathbf{0}}$. Thus the inclusion $\{\underline{\mathbf{0}}\} \hookrightarrow U_{\mathcal{A}_{n}}^{\mathbf{R}}$ induces an $S_{n}$-deformation retract to a one point space. Therefore, by standard arguments the map $U_{\mathcal{A}_{n}^{\text {col,s }}}^{\mathbf{R}} \rightarrow U_{\mathcal{A}_{n}^{\text {col,s }}}^{\mathbf{R}} / U_{\mathcal{A}_{n}}^{\mathbf{R}}$ defines a $S_{n}$-homotopy equivalence. Let $X_{n}$ be the closed simplicial cone $\mathbf{R} \times \mathbf{R}_{+}^{n-1} \cong\left\{\left(x_{1}, \ldots, x_{n}\right) \in \mathbf{R}^{n} \mid x_{1} \leq \cdots \leq x_{n}\right\}$. Then the space $U_{\mathcal{A}_{n}^{\text {col,s }}}^{\mathbf{R}} / U_{\mathcal{A}_{n}}^{\mathbf{R}}$ is a wedge of $n$ ! copies of $Y_{n}=\left(X_{n} \cap U_{\mathcal{A}_{n}^{\text {col,s }}}^{\mathbf{R}}\right) /\left(X_{n} \cap U_{\mathcal{A}_{n}}^{\mathbf{R}}\right)$. The $n$ ! spaces are permuted freely according to the regular representation, the image of $U_{\mathcal{A}_{n}}^{\mathbf{R}}$ serves as the wedge point. We already know by the non-equivariant part of Proposition 4.6 that $U_{\mathcal{A}_{n}^{\text {col,s }}}^{\mathbf{R}}$ is homotopic to a wedge of $(s-1) \cdots((s-1)+(n-1))$ spheres of dimension $n$ and no point in $Y_{n}$ is fixed by an element of $S_{n}$. From this it follows that $Y_{n}$ is homotopic to $\frac{1}{n !} \cdot(s-1) \cdots((s-1)+(n-1))$ spheres of dimension $n$.

Note, that in part (i) the conclusion that the cohomology is free and in part (ii) the conclusion that the union is homotopic to a wedge of spheres is known to be true in general for hyperplane arrangements (see [11 and [21]). 
As another immediate consequence we obtain a result on the cohomology of the complement of $\mathcal{A}_{n}^{\text {col,s }}$ in a rank one local system. We emphasize this otherwise standard application of the combinatorial methods here, since it fits in the framework of the considerations by Schechtman \& Varchenko 12 . Let $\mathcal{A}$ be some arrangement of affine complex hyperplanes in $\mathbf{C}^{n}$. Let $\xi=\left(\xi_{H}\right)_{H \in \mathcal{A}}$ be some vector of complex numbers. Then we denote by $\omega_{\xi}$ the differential form $\sum_{H \in \mathcal{A}} \xi_{H} \cdot \frac{d H}{H}$, where $H$ is identified with a linear form defining $H$.

Proposition 5.2 Let $1 \leq s, n$. Let $\xi=\left(\xi_{H}\right)_{H \in \mathcal{A}_{n}^{\mathrm{col}, \mathrm{s}}}$ be some vector of complex numbers, such that for all $V \in L_{\mathcal{A}_{n}^{\mathrm{col}, \mathrm{s}}} \backslash\{\hat{0}\}$ the sum $\sum_{H \leq V} \xi_{H}$ over all hyperplanes $H$ containing $V$ does not vanish. Then the rank of the cohomology $H^{i}\left(\mathbf{C}^{n} \backslash U_{\mathcal{A}_{n}^{\mathrm{col}, \mathrm{s}},} \mathcal{L}_{\omega_{\xi}}\right)$ of $\mathbf{C}^{n} \backslash U_{\mathcal{A}_{n}^{\text {col,s }}}$ with coefficients in the rank one local system $\mathcal{L}_{\omega_{\xi}}$ defined by $\omega_{\xi}$ is given by $(s-1) \cdots(s+n-2)$ for $i=n$ and 0 in all other dimensions.

Proof. By the work of Esnault, Viehweg \& Schechtman [6] it follows that under the given assumptions the cohomology with coefficients in the rank one local system vanishes except in dimension $n$. By general facts or by the work of Yuzvinsky [20] we have $\sum_{i \geq 0}(-1)^{i} \operatorname{rank}^{i}\left(\mathbf{C}^{n} \backslash U_{\mathcal{A}_{n}^{\text {col,s }}}, \mathcal{L}_{\omega_{\xi}}\right)=\chi\left(L_{\mathcal{A}_{n}^{\text {col,s }}}, 1\right)$. Hence, the assertion follows from Corollary 4.5.

Finally, we turn our interest to the quotient spaces $\left(\mathbf{C}^{n} \backslash U_{\mathcal{A}_{n}^{\mathrm{col}, \mathrm{s}}}\right) / S_{n}$. We recall a basic fact about symmetric products of complex lines.

Proposition 5.3 Let the symmetric group $S_{n}$ act on complex $n$-space $\mathbf{C}^{n}$ by permuting the coordinates. Then the map that sends an $n$-tuple $\left(z_{1}, \ldots, z_{n}\right)$ to the polynomial $f(X)=\left(X-z_{1}\right) \cdots\left(X-z_{n}\right)$ induces an homeomorphism from $\mathbf{C}^{n} / S_{n}$ to $\mathbf{C}^{n}$.

For Proposition 5.3 we immediately infer the following interpretation.

Lemma 5.4 The space $\left(\mathbf{C}^{n} \backslash U_{\mathcal{A}_{n}^{\text {cols }}}\right) / S_{n}$ is homeomorphic to the space of monic complex polynomials of degree $n$ with no double root and no root in the set $[s]$.

Using our description of the $S_{n}$-action on cohomology we obtain:

Theorem 5.5 Let $1 \leq s, n$.

(i) $\widetilde{H}^{1}\left(\left(\mathbf{C}^{n} \backslash U_{\mathcal{A}_{n}^{\text {col,s }}}\right) / S_{n}, \mathbf{Q}\right) \cong \mathbf{Q}^{s+1}$.

(ii) $\widetilde{H}^{i}\left(\left(\mathbf{C}^{n} \backslash U_{\mathcal{A}_{n}^{\text {col,s }}}\right) / S_{n}, \mathbf{Q}\right) \neq 0$ for $i=2, \ldots, n-1$. The rank is given by the number of $S_{n}$-orbits of elements $\left(\tau=B_{1}|\ldots| B_{t},\left(l_{1}, \ldots, l_{t}\right)\right)$ in $\Pi_{n}^{\text {col,s }}$ such that $l_{i}=0$ implies $\left|B_{i}\right|=1,2$ and there is at most one index $i$ such that $l_{i}=0$ implies $\left|B_{i}\right|=2$. 
Proof. In order to determine the rational cohomology of the quotient it suffices to determine the multiplicity of the trivial representation of $S_{n}$ in the cohomology of $\mathrm{C}^{n} \backslash U_{\mathcal{A}_{n}^{\text {col,s }}}$. Note, that in general for a $G$-space $X$, which is CW-complex and $G$ a finite group, the rational cohomology of $X / G$ is given by the space of $G$-invariants on the rational cohomology of $X$. We know by Proposition 3.1 and Corollary 3.2 that intervals $[\hat{0}, p], p \in \Pi_{n}^{\text {col,s }}$, are homotopic to a wedge of spheres of dimension $\operatorname{rank}(p)-2$. Let $V_{p}$ be the subspace in $L_{\mathcal{A}_{n}^{\text {col,s }}}$ corresponding to $p \in \Pi_{n}^{\text {col,s }}$. Then $\operatorname{codim}\left(V_{p}\right)=2 \operatorname{rank}(p)-$ note that we consider real codimension. Therefore, $[\hat{0}, p]$ contributes to the cohomology of $\mathbf{C}^{n} \backslash U_{\mathcal{A}_{n}^{\text {col,s }}}$ in dimension $\operatorname{rank}(p)$.

(i) There are exactly $s+1$ orbits of $S_{n}$ on elements of rank 1 in $\Pi_{n}^{\text {col,s }}$ satisfying the conditions of assertion (ii) - one orbit of partitions with one block of size two and no blocks colored, $s$ orbits of the partition $1|\cdots| n$ with exactly one block colored.

(ii) Let $p=\left(\tau=B_{1}|\cdots| B_{t},\left(l_{1}, \ldots, l_{t}\right)\right)$ be some element of $\Pi_{n}^{\text {col,s }}$. Let $G \cong S_{\left|B_{1}\right|} \times$ $\cdots \times S_{\left|B_{f-1}\right|} \times S_{e_{1}}\left[S_{1}\right] \times \cdots \times S_{e_{n}}\left[S_{n}\right]$ be the stabilizer of $p=\left(\tau,\left(l_{1}, \ldots, l_{t}\right)\right)$ in $S_{n}$. By Corollary 4.3 the $S_{n}$-character on $\operatorname{ind}_{G}^{S_{n}} \widetilde{H}_{n-t+f-2}\left(\left[\hat{0},\left(\tau,\left(l_{1}, \ldots, l_{t}\right)\right)\right]\right)$ is given by $\mathbf{r}_{\left|B_{1}\right|} \cdots \mathbf{r}_{\left|B_{f-1}\right|} \cdot \mathbf{s g n} \mathbf{e}_{e_{1}}\left[\pi_{1}\right] \cdot \mathbf{1}_{e_{2}}\left[\pi_{2}\right] \cdots$ for suitable parameters. Now, by the work of Sundaram [15, Corollary 2.3] the trivial $S_{n}$-representation appears in $\mathbf{r}_{\left|B_{1}\right|} \cdots \mathbf{r}_{\left|B_{f-1}\right|} \cdot \mathbf{s g n}_{e_{1}}\left[\pi_{1}\right] \cdot \mathbf{1}_{e_{2}}\left[\pi_{2}\right] \cdots$ if and only if $e_{2} \leq 1, e_{3}=\cdots=e_{n}=0$ and in this case it appears exactly once. But this condition translates immediately to the condition stated in assertion (ii). It is clear that such partitions exist for all ranks.

Now, for $s \rightarrow \infty$ the space $M_{\mathcal{A}_{n}^{\text {col,s }}}$ can be used to "approximate" the space of complex polynomials with no integral root. Note, that the combinatorics of $\mathcal{A}_{n}^{\text {col,s }}$ does not depend on the actual values of the excluded coordinates. Thus by choosing the sequence $0,-1,+1,-2,2, \ldots$ we exhaust the integers when $s \rightarrow \infty$.

\section{References}

[1] V.I. Arnol'd, The cohomology ring of the colored braid group, Math. Notes 5 (1969), 138-140.

[2] C.A. Athanasiadis, Characteristic polynomials of subspace arrangements and finite fields, (Preprint 1996).

[3] A. Björner and J.W. Walker, A homotopy complementation formula for partially ordered sets, European J. Combin. 4 (1983), 11-19.

[4] E. Brieskorn, Sur les groupes de tresses, Séminaire Bourbaki 1971/72 (Berlin, Heidelberg, New York), Lecture Notes in Math., vol. 317, Springer, Berlin, Heidelberg, New York, 1973, pp. 21-44. 


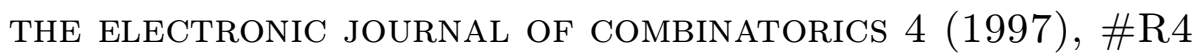

[5] P. Edelman and V. Reiner, Free hyperplane arrangements between $A_{n}$ and $B_{n}$, Math. Z. 215 (1994), 347-365.

[6] H Esnault, E. Viehweg, and V. Schechtmann, Cohomology of local systems of the complement of hyperplanes, Invent. Math. 109 (1993), 557-561.

[7] R. Fox and L. Neuwirth, The braid groups, Math. Scand. 10 (1962), 119-126.

[8] G.I. Lehrer and L. Solomon, On the action of the symmetric group on the cohomology of the complement of its reflecting hyperplanes, J. Algebra 104 (1986), 410-424.

[9] J.R. Munkres, Elements of algebraic topology, Addison-Wesley, Menlo Park, CA, 1984.

[10] P. Orlik and L. Solomon, Combinatorics and topology of complements of hyperplanes, Invent. Math. 56 (1980), 167-189.

[11] P. Orlik and H. Terao, Arrangements of hyperplanes, Grundlehren der mathematischen Wissenschaften, vol. 300, Springer, Berlin, Heidelberg, New York, 1992.

[12] V. Schechtmann and A. Varchenko, Arrangements of hyperplanes and Lie algebra homology, Invent. Math. 106 (1991), 134-194.

[13] R. P. Stanley, Some aspects of groups acting on finite posets, J. Combin. Theory Ser. A 32 (1982), 132-161.

[14] _ Enumerative combinatorics, I, Wadsworth \& Brooks/Cole, Monterey, CA, 1986.

[15] S. Sundaram, The homology representations of the symmetric group on CohenMacaulay subposets of the partition lattice, Adv. in Math. 104 (1994), no. 2, $225-296$.

[16] S. Sundaram and V. Welker, Group representations on the homology of products of posets, J. Combin. Theory Ser. A 73 (1996), 174-181.

[17] M. Wachs and J. Walker, Geometric semilattices, Order 4 (1986), no. 2, 367-387.

[18] V. Welker, Equivariant homotopy of posets and some applications to subgroup lattices, J. Combin. Theory Ser. A 69 (1995), no. 1, 61-86.

[19] _ Partition lattices, group actions on subspaces arrangements $\& 5$ combinatorics of discriminants, Habilitationsschrift, Universität Essen, 1996.

[20] S. Yuzvinsky, Cohomology of the Brieskorn-Orlik-Solomon algebras, Comm. Algebra 23 (1995), no. 14, 5339-5354.

[21] G. M. Ziegler and R. Živaljević, Homotopy types of subspace arrangements via diagrams of spaces, Math. Ann. 295 (1993), 527-548. 